\title{
Situación nutricional de niños de tres a cinco años de edad en tres distritos de Lima Metropolitana. Perú, 2016
}

Pedro Javier Navarrete Mejía ${ }^{1, a}$, Juan Carlos Velasco Guerrero ${ }^{1, a}$, Manuel Jesús Loayza Alarico ${ }^{1, a}$, Zoel Aníbal Huatuco Collantes ${ }^{1, \mathrm{~b}}$

\section{RESUMEN}

Objetivo: Conocer la situación nutricional de los niños de 3 a 5 años de edad, residentes en los distritos de Villa el Salvador, San Juan de Miraflores y San Juan de Lurigancho para el periodo comprendido entre abril y junio del 2016.

Materiales y métodos: Estudio prospectivo, transversal no experimental. La información comprende a 1416 niños y niñas de los distritos de Villa el Salvador, Villa María del Triunfo y San Juan de Lurigancho (340, 322 y 754 respectivamente). La información recogida comprendió datos de peso, talla y edad, en base a esta información se determinó desnutrición crónica, desnutrición aguda, sobrepeso y obesidad según el patrón de referencia de la Organización Mundial de la Salud. Se calculó los indicadores T/E y P/T.

Resultados: Se determinó que la población del distrito de San Juan de Lurigancho presentó mayor desnutrición crónica $(8.6 \%)$ y mayor sobrepeso $(11.0 \%)$, la población del distrito de Villa María del Triunfo presentó mayor desnutrición aguda (1.3\%) y la de Villa El Salvador mayor obesidad (4.6\%).

Conclusión: En los últimos años el tema nutricional tiene un gran impulso por parte del gobierno peruano a través de programas sociales, estando pendiente el enfoque sobre los determinantes a nivel de causalidad, hoy solo se mira la seguridad alimentaria, cuidado materno-infantil y calidad del entorno de salud descuidando el enfoque de causalidad, considerado como el más importantes del abordaje del problema. Los grandes lineamientos nacionales en materia de nutrición deben incluir estrategias no solo de reducción de los problemas nutricionales sino también prevención de estos.

Palabras clave: desnutrición crónica, desnutrición aguda, obesidad, sobrepeso.

\section{Nutritional status of children from three to five years old in three districts of Lima. Peru, 2016}

ABSTRACT

Objective: To know the nutritional status of children from 3 to 5 years old living in the districts of Villa el Salvador, San Juan de Miraflores and San Juan de Lurigancho for the period from April to June 2016.

Material and methods: Prospective, transversal, non-experimental study. The information includes 1,416 children from the districts of Villa El Salvador, Villa Maria del Triunfo and San Juan de Lurigancho (340, 322 and 754 respectively). The information collected included data on weight, height and age and based on this information, chronic malnutrition, acute malnutrition, overweight and obesity was determined according to the reference standard of the World Health Organization. S/A and W/S indicators was calculated.

Results: It was determined that the population of San Juan de Lurigancho had higher chronic malnutrition (8.6\%) and higher overweight (11.0\%), the population of the district of Villa Maria del Triunfo had higher acute malnutrition (1.3\%) and population of Villa El Salvador greater obesity $(4.6 \%)$.

Conclusion: In recent years the nutritional issue has a major boost by the Peruvian government through social programs, focusing on the determinants level of causality. Today only food security, maternal and child care and environmental quality are observed, neglecting health approach causality, considered the most important approach to the problem. Large national guidelines on nutrition strategies should include not only reduction of nutritional problems but also preventing these.

Key words: chronic malnutrition, acute malnutrition, obesity, overweight.

1. Centro de Investigación de Salud Pública, Instituto de Investigación, Facultad de Medicina Humana. Universidad de San Martín de Porres.

a. Doctor en Salud Pública.

b. Doctor en Medicina. 


\section{INTRODUCCIÓN}

La Encuesta Nacional de Demografía y Salud Familiar (ENDES) entre los años 2007 y 2015 permitió conocer que la desnutrición crónica (Patrón OMS) en niños menores de cinco años descendió de $28.5 \%$ a $14.4 \%$. Las tendencias en Lima Metropolitana corresponden a un escenario con prevalencias bajas de desnutrición crónica, llegando a 5.3\% al año $2015^{(1)}$. La problemática con la información del ENDES radica en que no permite inferenciar a nivel distrital y/o local; esta falta de información limita las acciones de seguimiento, monitoreo y evaluación del impacto de las intervenciones que realiza el estado en materia nutricional ${ }^{(2,3)}$.

Malnutrición se define como deficiencia, exceso o desequilibrio de nutrientes, la malnutrición es la causa de efectos adversos en la composición corporal, la función y el estado clínico de los individuos ${ }^{(4)}$. Un niño malnutrido no solo tendrá un deficiente crecimiento, sino también un mayor riesgo de morbididad y mortalidad cardíaca, renal ósea.

La malnutrición en sus extremos es conocida como desnutrición y obesidad ${ }^{(5)}$. El padecer de malnutrición es una limitante en la productividad de los individuos y está estrechamente ligada a una mayor demanda de servicios sanitarios con una impostergable carga económica para él, la familia y el Estado ${ }^{(6)}$. En los niños esta situación es el resultado de dietas pobres en vitaminas y minerales; otro factor importante a tener en cuenta es la presencia de enfermedades infecciosas asociadas a condiciones de vivienda y ausencia de servicios básicos ${ }^{(7,8)}$.

En los últimos años, nuestro país ha mostrado importantes avances en la reducción de la malnutrición, en especial de la desnutrición crónica infantil; sin embargo, aun cuando se ha alcanzado el objetivo de desarrollo del milenio del 2015, las inequidades aún se evidencian a nivel regional y en zonas de pobreza $^{(1,9,10)}$.

Otro aspecto importante en la población infantil es el sobrepeso y la obesidad siendo la población infantil uno de los grupos de mayor riesgo; la Encuesta Nacional de Hogares del 2008 muestra evidencia concluyente que la obesidad y el sobrepeso son un problema principalmente concentrado en zonas urbanas como Lima $(11.4 \%){ }^{(11)}$.

La investigación incluyó a los distritos de Villa El Salvador, Villa María del Triunfo y San Juan de
Lurigancho por ser los distritos de mayor pobreza en el marco de Lima Metropolitana, según el Mapa de Pobreza distrital elaborado por el Instituto Nacional de Estadística e Informática del Perú ${ }^{(12)}$.

El objetivo del estudio fue conocer la situación nutricional de los niños de 3 a 5 años de edad residentes en los distritos de Villa el Salvador, San Juan de Miraflores y San Juan de Lurigancho para el periodo comprendido entre abril y junio del 2016, con la finalidad de disponer de información local que contribuya a tomar decisiones orientadas a mejorar el estado nutricional de la población más vulnerable.

\section{MATERIAL Y MÉTODOS}

Estudio prospectivo, transversal no experimental. La información comprendió a 1416 niños y niñas de 3 a 5 años residentes en los distritos de Villa el Salvador (340 niños), Villa María del Triunfo (322 niños) y San Juan de Lurigancho (754 niños).

La información recogida de los niños y niñas comprendió datos de peso, talla y edad. En base a esta información se determinó desnutrición crónica, desnutrición aguda, sobrepeso y obesidad, se hizo uso de los criterios según el patrón de referencia de la Organización Mundial de la Salud. Los niños y niñas fueron evaluados en los nidos públicos (instituciones educativas) de las jurisdicciones distritales previamente determinadas. El proceso de tallar y pesar a los niños fue supervisado por profesionales capacitados y con la experiencia requerida para dicho proceso. Se calculó los indicadores T/E y P/T.

La evaluación formó parte del trabajo de campo de los estudiantes de enfermería de una universidad privada de Lima. Se han respetado escrupulosamente los criterios bioéticos mediantes el consentimiento informado de los padres de familia.

Los datos fueron recolectados entre abril y junio del 2016.

\section{RESULTADOS}

La evaluación de los niños y niñas, con edades que fluctúan entre los 3 y 5 años, mediante los indicadores T/E y P/T permitió conocer su situación nutricional (Patrón OMS). La evaluación se realizó en 1416 niños y niñas de 3 a 5 años (Tabla 1 ). 
Pedro Javier Navarrete Mejía, Juan Carlos Velasco Guerrero, Manuel Jesús Loayza Alarico, Zoel Aníbal Huatuco Collantes

Tabla 1. Población de niños y niñas que participó en la investigación

\begin{tabular}{|l|c|}
\hline \multicolumn{1}{|c|}{ Distrito } & Niños y niñas evaluados \\
\hline Villa el Salvador & 340 \\
\hline Villa María del Triunfo & 322 \\
\hline San Juan de Lurigancho & 754 \\
\hline Total & 1413 \\
\hline
\end{tabular}

Las mediciones permitieron determinar que los niños y niñas del distrito de San Juan de Lurigancho presentaron una mayor prevalencia de desnutrición crónica (8.6\%) en comparación con los niños de Villa María del Triunfo y Villa El Salvador (7.3\% y 6.9\% respectivamente). En el caso de la desnutrición aguda como estado nutricional los niños y niñas del distrito de Villa María del Triunfo presentaron mayor prevalencia (1.3\%) en relación a los niños y niñas de los distrito de Villa El Salvador y San Juan de Lurigancho ( $0.9 \%$ en ambos casos) (Tabla 2 ).

Tabla 2. Desnutrición crónica y aguda por distritos

\begin{tabular}{|l|c|c|}
\hline \multicolumn{1}{|c|}{ Distrito } & Desnutrición crónica & Desnutrición aguda \\
\hline Villa el Salvador & $6.9 \%$ & $0.9 \%$ \\
\hline Villa María del Triunfo & $7.3 \%$ & $1.3 \%$ \\
\hline San Juan de Lurigancho & $8.6 \%$ & $0.9 \%$ \\
\hline
\end{tabular}

En cuanto al sobrepeso y obesidad se pudo determinar que para el primer caso los niños y niñas del distrito de San Juan de Lurigancho presentan mayor prevalencia (11.0\%) con relación a Villa María del Triunfo y Villa El Salvador $(9.1 \%$ y $10.8 \%$ respectivamente), y para la obesidad es el distrito de Villa el Salvador el que presenta la mayor prevalencia (4.6\%) (Tabla 3).

Tabla 3. Sobrepeso y obesidad por distritos

\begin{tabular}{|l|c|c|}
\hline \multicolumn{1}{|c|}{ Distrito } & Sobrepeso & Obesidad \\
\hline Villa el Salvador & $10.8 \%$ & $4.6 \%$ \\
\hline Villa María del Triunfo & $9.1 \%$ & $4.2 \%$ \\
\hline San Juan de Lurigancho & $11.0 \%$ & $4.5 \%$ \\
\hline
\end{tabular}

\section{DISCUSIÓN}

Hoy se conoce que las alteraciones en el estado nutricional impactan directamente sobre el desarrollo cognitivo de los niños con efectos adversos sobre el proceso de aprendizaje y el rendimiento escolar a corto y mediano plazo ${ }^{(4,5,13)}$.

No soslayemos la importancia del estado nutricional como defensa frente a las enfermedades ya que, alteraciones del estado nutricional incrementan la morbilidad y la mortalidad en la niñez. La evidencia científica demuestra los efectos negativos de un mal estado nutricional sobre todo en las primeras etapas de vida. Problemas nutricionales incrementan el riesgo de padecer enfermedades crónicas (diabetes, enfermedades cardiovasculares, entre otras) y menores oportunidades para alcanzar logros académicos que traerán como consecuencia menores ingresos económicos en la adultez ${ }^{(14,15)}$.

La desnutrición crónica se define como el estado en el cual los niños y niñas tienen baja estatura para su edad (T/E) con relación a una población de referencia, y no es otra cosa que el reflejo de una periódica e inadecuada alimentación o ingesta insuficiente de nutrientes, claro que también puede ser consecuencia de múltiples episodios de enfermedades diarreicas o respiratorias principalmente. 
La desnutrición crónica es un indicador de desarrollo del país y su disminución contribuirá a garantizar el desarrollo de la capacidad física, intelectual, emocional y social en la primera etapa de vida ${ }^{(16)}$.

Tener un $\mathbf{8 . 6 \%}$ de desnutrición crónica en los niños y niñas del distrito de San Juan de Lurigancho, $7.3 \%$ en Villa María del Triunfo y $6.9 \%$ en Villa El Salvador es preocupante, por lo expuesto líneas arriba, tendríamos un alto porcentaje de peruanos - para los distritos y población en estudio - destinados a problemas en su capacidad física, intelectual y social.

El promedio de desnutrición crónica para Lima Metropolitana durante el 2015, según el Instituto de Gestión de servicios de Salud, en niños y niñas de 36 a 47 meses fue de $7.8 \%$ y para los de 48 a 59 meses fue de $6.0 \%$, si bien es cierto la cifra ha venido disminuyendo desde el 2011, no deja de ser preocupante por las implicancias futuras como actores importantes en el desarrollo del país ${ }^{(17)}$.

La desnutrición aguda se define como el estado en el cual los niños y niñas tienen un peso menor al esperado para su talla con relación a una población de referencia. Esta alteración es un condicionante de riesgo para la vida del menor. La desnutrición aguda es el reflejo de una reciente carencia en la ingesta de alimentos o nutrientes, y en algunos casos presencia de enfermedades agudas como las diarreas o enfermedades respiratorias. Como consecuencia de esta carencia de nutrientes las reservas energéticas se metabolizan para compensar las necesidades del organismo, resultando en una pérdida de peso debido a la desaparición de masa muscular y tejido graso. La pérdida de masa muscular y de tejido graso disminuyen la probabilidad de sobrevivir ${ }^{(18)}$.

La investigación determinó que los niños y niñas del distrito de Villa María del Triunfo presentan desnutrición aguda versus un $0.9 \%$ en los distritos de Villa El Salvador y San Juan de Lurigancho. Para la Organización Mundial de la Salud se establece como un problema de salud pública cuando la prevalencia de desnutrición aguda es mayor a 20\%, para la población estudiada no se llega a esa magnitud, pero no deja de ser preocupante puesto que evidencia un problema de nutrición que debe ser abordado inmediatamente. Para el año 2015 el Instituto de Gestión de Servicios de Salud determinó la prevalencia de desnutrición aguda en los niños y niñas menores de 5 años de Lima metropolitana en $0.9 \%{ }^{\left({ }^{(17)}\right.}$.
Las alteraciones del estado nutricional no solo se dan por déficit sino también en valores sobre los niveles normales, denominándose sobrepeso y obesidad.

Para el 2015 los niños y niñas menores de 5 años de Lima metropolitana, según el reporte del Instituto de Gestion de Servicios de Salud, sitúa el sobrepeso en $10 \%$ y la obesidad en $3.8 \%{ }^{(18)}$.

La prevalencia para la población estudiada en sobrepeso y obesidad para el distrito de Villa El Salvador es $10.8 \%$ y $4.6 \%$, para San Juan de Lurigancho $11.0 \%$ y $4.5 \%$, y para Villa María del Triunfo $9.1 \%$ y $4.2 \%$ respectivamente. Esto es preocupante, por lo que la prevención del sobrepeso y la obesidad debe ser una estrategia prioritaria en salud nutricional. Al igual que la desnutrición, no debe perderse el enfoque multidisciplinario de su abordaje, debe incluirse el microambiente del menor (familia y escuela) y su macroambiente (autoridades, empresas, etc.).

El tema nutricional, si bien es cierto, viene teniendo gran impulso por parte del gobierno a través de programas sociales, no debe perderse de vista el enfoque sobre los determinantes a nivel de causalidad, debe enfocarse en la ingesta alimenticia y el estatus de salud (inmediata); seguridad alimentaria, cuidado materno-infantil y calidad del entorno de salud (subyacente); y disponibilidad de recursos económicos y entorno político (básica). Hoy como país nos concentramos principalmente sobre los determinantes subyacentes.

Debemos empezar a tener una visión más amplia del problema. La educación de la madre tiene un impacto directo sobre la nutrición de sus hijos, recordemos que el estudio se realizó en los tres distritos más pobres de Lima Metropolitana, posiblemente son los distritos donde las madres tienen menor grado de instrucción. Los grandes lineamientos nacionales en materia de nutrición deben incluir la participación social (actores y organizaciones de base); por otra parte debemos pasar de estrategias nacionales para reducir la desnutrición, la obesidad y el sobrepeso, a la formulación de políticas públicas enmarcadas en su no aparición.

\section{REFERENCIAS BIBLIOGRÁFICAS}

1. Pasricha S-R, Drakesmith H, Black J, Hipgrave D, Biggs B-A. Control of iron deficiency anemia in low- and middle-income countries. Blood.2013;121(14):2607-2617

2. INEI. Encuesta Demográfica y de Salud Familiar. Perú. 2012

3. INEI. Encuesta Demográfica y de Salud Familiar. Perú. 2015

4. Prakash S. Malnutrition and undernutrition. Medicine.2003; 43(2):18-22 
Pedro Javier Navarrete Mejía, Juan Carlos Velasco Guerrero, Manuel Jesús Loayza Alarico, Zoel Aníbal Huatuco Collantes

5. FAO. Información de seguridad alimentaria para la acción. Conceptos y Marcos de Seguridad Alimentaria. Información sobre seguridad alimentaria para la acción. 2010 [Internet].Recuperado en octubre de 2016 de http://www.fao.org/elearning/course/FC/es/pdf/trainerres ources/learnernotes0531.pdf

6. Colditz GA. Economic costs of obesity. Am J Clin Nutr.1992;55(2):503S-507S

7. Balarajan Y, Ramakrishnan U, Özaltin E, Shankar A, Subramanian S. Anaemia in low-income and middle-income countries. Lancet.2011;378(9809):2123-2135

8. WHO. Micronutrient deficiencies [Internet]. WHO. Recuperado en julio de 2016 de http://www.who.int/ nutrition/topics/ida/en/

9. Zimmermann MB, Hurrell RF. Nutritional iron deficiency.Lancet.2007;370(9586):511-520

10. Black D. Maternal and child undernutrition and overweight in low-income and middle-income countries. Lancet. 2013; 382: 427-451

11. INEI. Encuesta Nacional de Hogares sobre condiciones de vida y pobreza. Perú. 2008

12. INEI. Mapa de pobreza provincial y distrital 2013.Perú

13. Black R, Allen L, Bhutta Z, Caulfield L, De Onis M, Ezzati M, et al. Maternal and child undernutrition: global and regional exposures and health consequences. Lancet,Series Maternal and Child Undernutrition. 2008: 5-22

14. Victoria C, Adair L, Fall C, Hallal P, Martorell R, Ritcher L, et al. Maternal and child undernutrition: consequences for adult health and human capital. Lancet, Series Maternal and Child Undernutrition.2008: 23-40
15. WHO. WHO Child Growth Standards: Methods and development. Geneva.2006

16. INEI. Mapa de Desnutrición Crónica en Niños Menores de Cinco Años a Nivel Provincial y Distrital, 2007. Lima. 2009

17. IGSS. Análisis del estado nutricional de niños y gestantes que acuden a los establecimientos de salud en los distritos de Lima Metropolitana según el sistema de información del estado nutricional (SIEN), 2015. Recuperado en octubre de 2016 de http://igss.gob.pe/portal/files/ANALISIS_DEL_ESTADO_NUTRI CIONAL_SIEN_2015.pdf

18. Tazza R, Bullón L. ¿Obesidad o desnutrición? Problema actual de los niños peruanos menores de 5 años. An Fac Med. 2006;67(3):214-223

Fuentes de financiamiento:

Este artículo ha sido financiado por los autores.

Conflictos de interés:

Los autores declaran no tener ningún conflicto de interés.

\section{Correspondencia:}

Pedro Javier Navarrete Mejía

Dirección: Av. Alameda del Corregidor 1531, Urb. Los Sirius, Las Viñas, La Molina, Lima 12.

Teléfono: 998998844

Correo electrónico: pnavarretem@usmp.pe

Recibido: 12 de agosto de 2016

Aprobado: 10 de noviembre de 2016 